\title{
Historia y formación del profesorado
}

\author{
Valentín Martínez-Otero Pérez \\ Universidad Complutense de Madrid (Espanha) \\ Marlúcia Menezes de Paiva \\ Universidade Federal do Rio Grande do Norte (Brasil)
}

\section{Resumen}

En este artículo se realiza, en una primera parte, un repaso histórico de la educación en Brasil, en que se destaca la intervención de los poderes públicos tanto en la educación, en general, como en la formación del profesorado, en particular. Las iniciativas y reformas educativas revelan avances en el proceso modernizador, pero insuficientes en lo que se refiere a los sectores más desfavorecidos. La segunda parte del artículo se dedica, con carácter general, a aspectos pedagógicos y didacticos de esa formación inicial y permanente del profesorado. Se pone el foco en la descripción de cuatro modelos que, aunque condicionados por el pasado, aportan claves valiosas para la educación real.

Palavras-chave: Historia. Formación del profesorado. Modelos.

\section{História e formação docente}

\section{Abstract}

Na primeira parte deste artigo, se realiza uma revisão histórica da educação no Brasil, em que se destaca a intervenção dos poderes públicas na educação, tanto no ensino em geral como na formação dos professores em particular. Essas iniciativas e reformas educacionais revelam avanços no processo de modernização, mas insuficientes no que diz respeito aos setores mais desfavorecidos. A segunda parte do artigo é dedicada, em caráter geral, a aspectos pedagógicos e didáticos da formação inicial e continuada dos professores. O foco ocorre na descrição de quatro modelos que, embora condicionados pelo passado, proporcionam chaves valiosas para uma educação atual.

Keywords: História. Formação de professores. Modelos. 


\section{History and teacher education}

\section{Resumen}

In the first part of this article, we do a historical review of education in Brazil, in which we highlight the intervention of public authorities in education, both in general education as in teacher formation in particular. These educational initiatives and reforms reveal progress in the modernization process, but not enough for the most disadvantaged sectors. The second part of the article is devoted, in general, to pedagogical and didactic aspects of the initial and continuing teacher formation. The focus is on the description of four models that, although conditioned by the past, provide valuable keys to a current education.

Palabras clave: History. Teacher formation. Models.

\section{Introducción}

Nos adentramos en este artículo en la consideración histórica de la formación de los educadores, con la vista puesta en la mejora de su preparación teórico-práctica en nuestros días. En la actualidad, se discute mucho sobre el papel de la escuela como ámbito de formación del profesorado y, en consecuencia, también surge la controversia sobre el proyecto político-pedagógico y los saberes implicados en esa formación. Para respaldar nuestro estudio tomamos como referencia inicial de análisis la reconstitución histórica de esa formación, en la que se advierten contradicciones, continuidades e innovaciones.

En la segunda parte del texto, se abordan aspectos pedagógicos y didácticos del proceso formativo docente. Con arreglo a nuestras investigaciones las cuestiones planteadas en este trabajo necesitarían complementarse con una comparativa sobre formación del profesorado en Brasil y en España, lo que se deberá realizar durante nuestra etapa de investigación.

Así pues, se inicia el artículo con un recorrido histórico sobre la formación de profesores en Brasil, uno de los países de nuestra investigación. Se analizan después, con carácter general, aspectos pedagógicos y didácticos de esa formación inicial y permanente del profesorado. Por ello, nos detenemos en la revisión de modelos o esquemas teóricos de alcance práctico y que tienen proyección internacional. Se trata de orientaciones formativas que responden a tradiciones diferentes, a veces enfrentadas, y que proporcionan claves de acción docente igualmente desemejantes. Al respecto, cabe 
plantearse si los modelos son de tan distinta naturaleza que se hace inviable la complementariedad entre ellos o si, por el contrario, es posible y necesaria una cierta aproximación de planteamientos que permita, en el terreno práctico, una integración sinérgica. Algunos de estos modelos ponen el énfasis en los aspectos técnicos mientras que otros se interesan más por la condición personal del profesional, por su desenvolvimiento, por la reflexión sobre la propia práctica o por el carácter emancipador de la educación. En el ámbito de la actividad educativa, la diversidad de modelos se deja sentir en la concepción del proceso de enseñanza-aprendizaje, en el estilo educativo, en la organización de las clases, en el currículo etc. Al respecto, se ofrece una descripción de cuatro grandes modelos que, aunque no contempla todas las perspectivas teóricas existentes, ofrece una panorámica de la formación de los profesionales de la educación, sobre la que es preciso seguir investigando para desvelar y enriquecer muchos de sus aspectos concretos.

Queda claro, en definitiva, que los modelos formativos del pasado impactan en el presente y condicionan el futuro. Por ello, y sin obviar la necesaria contextualización, los cuatro modelos de formación del profesorado sumariamente presentados constituyen otras tantas miradas pedagógicas que responden a formas distintas de entender la educación y la profesión docente. En la medida en que estos modelos articulan teoría y práctica influyen con fuerza en diversos aspectos de la profesionalización docente y de la educación en su conjunto. La reflexión sobre los mismos se torna necesaria y cabe pensar que desde una actitud saludablemente integradora los cuatro aportan claves valiosas para la educación de nuestros días, repleta de dificultades, y para afrontar con esperanza, pese a las incertidumbres, los muchos desafíos, viejos y nuevos, que se presentan a nuestros países.

\section{Formación de profesores en Brasil}

Discurriendo sobre modelos formativos, emprendemos, inicialmente, una mirada a la Historia y la Formación de Profesores en Brasil, lo que nos lleva a situar históricamente cómo se contempló esta formación en las políticas públicas iniciadas en dicho país, en el proceso de su constitución como Estadonación. Con arreglo a esta afirmación, en la construcción de esta trayectoria, hemos optado por historizar las políiticas educacionales del Estado brasileño y 
las diversas reformas del sistema educativo en el proceso de constitución de un sistema de enseñanza, especialmente en la formación de profesores.

Elegimos llevar a cabo esta reconstitución histórica de la formación del profesorado en Brasil, tomando como marco inicial el período imperial, momento político que se extiende desde 1822 hasta el 15 de noviembre de 1889, comienzo de la República.

En términos políticos, de Estado-nación, se puede decir que el Estado brasileño comenzó en 1808 con la llegada de D. João VI, la familia real y la corte portuguesa, que huían de las tropas francesas de Napoleón Bonaparte. La llegada del Príncipe-Regente D. João y todo el aparato militar, administrativo y cultural de Portugal desencadenó el proceso de reformulación de los diversos aspectos de las políticas gubernamentales brasileñas, adequando-as às normas vigentes para la educación del gobierno portugués.

Com a independencia de Portugal, em 1822, el país, ante los rumbos, se adentraba en las prácticas definitorias del mundo moderno, civilizado y culto, esperado por muchos. No debe olvidarse, sin embargo, que quedó limitado a unos pocos lugares o, más bien, a pequeños círculos de personas: las élites nacionales instruidas. La mayor parte del país (casi el 80\% de la población) seguía siendo analfabeta y atrasada, muy alejada del ideal desarrollado, moderno y científico declarado.

En cuanto a la creación de cursos para la formación del profesorado, no hay ninguna referencia.

En cuanto a la legislación en materia de educación, por no hablar de las leyes provinciales, el Imperio nos legó:

a) La Constitución otorgada el 11 de diciembre de 1823, la primera Constitución brasileña, que estipulaba en su artículo 179: "la instrucción primaria gratuita para todos los ciudadanos".

b) Como resultado de la Constitución de 1824, el 15 de octubre de 1827, se promulgó la primera Ley General de Educación en el Imperio de Brasil, cuyo proyecto final determinó algunos cambios importantes, como se establece en el artículo 1: "En todas las ciudades, villas y lugares más poblados habrá las escuelas de primeras letras que sean necesarias". $\bigcirc$ cuando innovó en el Artículo 1 1, al decir: "Habrá escuelas de niñas en las ciudades, villas y lugares más poblados donde los presidentes provinciales, en consejo, consideren conveniente este establecimiento" (Lei de 15 de outubro de 1827). Se 
trataba de la instrucción pública que instituía las Escuelas de Primeras Letras. "Enel artículo 7 se establece que "Os que pretenderem ser providos nas cadeiras serão examinados publicamente legal perante os Presidentes, em Conselhos; e estes proverão o que fôr julgado mais digno e darão parte ao Governo para sua legal nomeação".

La misma Ley, instituye la enseñanza mutua, pero pronto demuestra su imposibilidad. Delega en los maestros que no conocen este método para que vayan a la capital a instruirse y se lo paguen ellos mismos. El gobierno no asume la formación de profesores, delega en los maestros la tarea de llevar a cabo su formación, en un momento histórico en el que conocemos las dificultades inherentes a un país en formación, económicamente subdesarrollado y sin dar la debida importancia a la Educación, porque ni siquiera había cursos de formación docente.

¿Cómo podrían estos maestros capacitarse a sí mismos? Es una gran interrogación.

c) Otra acción importante en el período Imperial, durante la Regencia, fue la promulgación del Acta Adicional de 1834, a la Constitución de 1824, que establece la descentralización de la educación, delegando el poder de legislar sobre la instrucción pública a las Asambleas Legislativas Provinciales, para los niveles primario y secundario. Por su parte, la educación superior, en general, y la organización general del Municipio Neutral quedaron bajo la responsabilidad de la administración nacional, hoy federal. Esa Acta, a diferencia de la Ley anterior, representó una fragmentación y un freno al desarrollo de la educación brasileña, ya que la mayoría de las provincias no tenían recursos y sus administradores no mostraron mayor compromiso con la educación, lo que se tradujo en una falta absoluta de escuelas para la población. Sólo el Municipio Neutral resultó beneficiado. Según Fernando de Azevedo:

[...] la educación tendría que arrastrarse a lo largo de todo el siglo XIX, desorganizada, anárquica, incesantemente desagregada. Entre la educación primaria y secundaria no hay puentes ni uniones: son dos mundos que están orientados, cada uno en su dirección. [...] No hay ninguna conexión, no solo verticalmente, entre los diversos grados de la jerarquía, tampoco horizontalmente, entre las mismas unidades escolares que operan lado a lado (AZEVEDO, 1996, p. 556-557). 
Esta situación benefició a la educación secundaria privada que alcanzó un gran desarrollo en el período, particularmente en las capitales. Surgieron colegios de sacerdotes lazaristas, jesuitas, franciscanos y otros, a los que asistieron los hijos de las élites brasileñas. Dentro de la educación secundaria oficial se crearon algunas instituciones, como los Liceos en las Provincias y el Colegio Pedro II, en 1837, en la Corte. El Colegio Pedro II sirvió como modelo institucional de humanidades, además de ser una referencia de enseñanza para las demás instituciones.

Finalmente, ha de mencionarse la creación de las Escuelas Normales por la falta de profesores para atender a la enseñanza en expansión, particularmente después de la promulgación de la Ley Imperial de 1827. Las primeras Escuelas Normales aparecieron en Niterói (1 835), Bahía (1 836), Ceará (1845) y São Paulo (1846). En general, estas Escuelas no tenían un funcionamiento regular: abrían y cerraban las puertas con mucha facilidad. Faltaban recursos, faltaban alumnos.

Con esta situación educativa, llegamos al final del Imperio en 1889, con aproximadamente el 82,6\% (IBGE, 2003) de la población analfabeta. El país experimentó algunas modificaciones con la independencia. Sin embargo, a pesar de muchas discusiones y debates, no se hizo casi nada concreto por la instrucción pública y, en consecuencia, por la formación de maestros.

\section{La república y la institucionalización de la escuela}

Al principio, la proclamación de la República en 1889 no produjo grandes cambios desde el punto de vista educativo. En términos políticos, cambió el Gobierno Federal, en el cual tres poderes armónicos, en teoría, dirigían la nación: el ejecutivo, el legislativo y el judicial. El poder moderador, exclusivo del emperador, se extinguió.

En términos educativos, se asiste al inicio de los debates e intentos de organizar un sistema educativo. La influencia positivista, habitual en los intelectuales, políticos y militares, aumentó la discusión sobre la importancia de modificar la educación nacional, principalmente para atender al mundo capitalista, racional y tecnológico que se consolidó en todo Occidente. La educación se promocionó como el marco estructurante y favorecedor de ese mundo nuevo y moderno que se estaba desarrollando. 
Se observa en las primeras décadas del siglo XX un discurso de sobrevaloración de los efectos de la educación en la transformación de la nación. Por la educación se alcanzaría el nivel propio de los países civilizados. Por la educación se conquistaría la identidad nacional aún lejana. Sin embargo, las pautas para esta nueva escuela, que impregnó estos discursos, brindaron a los educadores experiencias educativas en los Estados que demostraron una nueva forma de enseñar (PAIVA, 2009).

Con la República surgió la institución escolar que sería el modelo para la concepción de lo que sería la escuela moderna: el grupo escolar, que actuaría en la consolidación de un nuevo proceso civilizatorio. Esta escuela sería considerada como el elemento racional, modernizador y organizador de la sociedad, haciéndola más justa e integrada con los parámetros modernos del progreso social y, quizás, económico.

Francinaide de Lima Silva (2009, p.29), en su tesis de maestría que tuvo como objeto de estudio el Grupo Escolar Modelo Augusto Severo, en Natal (RN), afirma que estas escuelas, nacidas al comienzo de la República, surgieron como concepción de escuela moderna y representan "[... ] la génesis de la escuela pública primaria en el país, dotada de un espacio y unas prácticas de una institución educativa formal [...] que se basó en la distribución homogénea de los estudiantes, en la existencia de varias aulas y en un mayor número de maestros ". Con los grupos escolares, el país adoptaba el modelo de una escuela moderna y pasaría a necesitar profesores con nivel formativo adecuado a esta nueva escuela.

En las primeras décadas del siglo XX, la economía en Brasil dio pasos significativos. La década de 1920 fue prodigiosa en innovaciones, en movimientos culturales y políticos. Tuvo lugar la Semana del Arte Moderno, en São Paulo, que permitió a los artistas hablar de los cambios en su sector; los educadores crearon la Asociación Brasileña de Educación (ABE), que promovió las notorias Conferencias de Educación Brasileñas (CBE); se creó el Partido Comunista; los militares se organizaron en el denominado movimiento de tenentismo; la Iglesia Católica también organizó sus marcos intelectuales, mediante la creación de periódicos, revistas y el importante Centro Dom Vital, que buscaba dar carácter orgánico a las prácticas educativas y evangelizadoras de la Iglesia. Esta es una imagen que muestra signos de insatisfacción en las clases medias, una estampa que revela cambios. 
Incluso en ese momento histórico, se produjo la crisis mundial del capitalismo en 1929, lo que provocó una debacle en la economía mundial y, en consecuencia, en la economía brasileña; las élites tradicionales eran frágiles. Canalizando esas insatisfacciones surgió un movimiento que pasó a la historia con la denominación de Revolución de los 30 y permitió cambios de los planes de poder de las élites que gobernaban el país.

En medio siglo de la República, se produjo poca transformación en el marco educativo. Con respecto a la educación, las tasas continuaron mostrando altas tasas de analfabetismo, ya que, en 1890, según el censo del IBGE, la tasa de analfabetismo en Brasil era del 82,6\%, en 1920 del $71,2 \%$ y en 1940 del 61,2\% (FERRARO, 2009). Si observamos la perspectiva relativa, basada en el año 1890, veremos una reducción del 11,2\% en el analfabetismo hasta 1940. Pero si observamos a la población en términos absolutos, el país mostró un gran aumento en el número de personas analfabetas: 10,1 millones en 1890, hasta alcanzar el nivel de poco más de 21,3 millones de personas analfabetas en 1940, a la edad de 5 años o más. Eso retrata la desconexión de los gobernantes con la educación, a pesar de los discursos que mostraron la importancia de la educación en el nuevo momento de civilidad, ciudadanía 8 y modernidad que se pretendía lograr.

El gobierno establecido en Brasil en la década de 1930 se esforzó para organizar las escuelas en base a criterios centralistas, lo cual se constató con la creación de un Ministerio de Educación y Salud. Por primera vez el país tenía un ministerio exclusivo para educación y salud.

La nueva administración elaboró dos reformas educativas importantes. La primera, en 1931, Reforma Campos, se dirigió principalmente a organizar la educación secundaria y la educación superior. La segunda, en el período dictatorial del Estado Nuevo, la Reforma Capanema, que consistió en la preparación de leyes llamadas Leyes Orgánicas de Enseñanza, que cubrían todos los niveles de enseñanza, incluida una Ley Orgánica de Enseñanza Normal, el 2 de enero de 1946, que se ocupó de la formación del professorado. Sin embargo, esta Ley mantuvo la dualidad de esta formación, con la existencia de dos ciclos: o primero ciclo, de cuatro años de duración, y el segundo, con tres años de duración. El objetivo del primer ciclo era capacitar a los regentes de la escuela primaria y trabajar en las escuelas regionales normales. El segundo ciclo, que duró tres años, fue diseñado para capacitar a maestros de primaria y funcionaría en escuelas normales e institutos de educación. Estos, además 
de los cursos mencionados anteriormente, tendrían un jardín de infantes y una escuela primaria adjuntos y también ofrecerían cursos de especialización para maestros de primaria en las áreas de educación especial, educación superior, dibujo y artes aplicadas, música y canto, y cursos de administrador escolar para capacitar directores, consejeros e inspectores escolares. Estos institutos de educación dieron lugar a cursos de pedagogia de nível superior. La universidad del Distrito Federal y la Universidad de São Paulo fueron pioneras em este cambio. La enseñanza dirigida a la formación del profesorado finalmente se estaba delineando, en el nivel médio y ahora comenzando em el nivel superior.

La próxima reforma educativa, la Ley de Directrices y Bases de la Educación, Ley $n^{\circ}$ 4024, de 20 de diciembre de 1961, apareció en el contexto posterior a la Segunda Guerra Mundial y, aunque trajo propuestas más democráticas, sin cambios importantes, tuvo poca vigencia, porque en abril de 1964 hubo un golpe de Estado que llevó al país a una dictadura civil-militar. El grupo que tomó el poder buscó crear sus leyes en el contexto apropiado de un estado totalitario.

La nueva reforma, la Ley $n^{0}$ 5.692, de 11 de agosto de 1971, creó una nueva estructura para la enseñanza de primer y segundo grado. Para la educación superior, hubo una reforma en 1969. En esta nueva estructura, en la Ley 5692, las Escuelas Normales desaparecieron. En su lugar, se instituyó una habilitación específica para el magisterio, en el nivel de segundo grado. Esta habilitación se organizó en dos modalidades básicas: una con una duración de tres años (2.200 horas), que permitiría la enseñanza hasta el cuarto grado; y otra con una duración de cuatro años (2.900 horas), que permifía el magisterio hasta la sexta serie del primer grado. El plan de estudios mínimo comprendía el núcleo común, obligatorio en todo el territorio nacional para toda la enseñanza de $1^{\circ}$ y $2^{\circ}$ grado, con objeto de garantizar la formación general; y una parte diversificada, dirigida a la formación especial.

El curso normal anterior dio paso a una habilitación de segundo grado. La formación de docentes para la antigua enseñanza primaria se redujo así a una habilitación dispersa entre muchas otras, lo que constituyó una situación precaria muy preocupante. Para las últimas cuatro series de enseñanza de primer grado y para la enseñanza de segundo grado la ley $n^{\circ} .5 .692$, de 11 de agosto de 1971, previó la formación de docentes de nivel superior, en cursos de licenciatura cortos ( 3 años) o completos (4 años), el curso de pedagogía. Este curso, además de capacitar profesores para la habilitación 
específica del Magisterio, se confería la atribución de formar a los especialistas en Educación, incluidos directores de escuelas, orientadores educativos, supervisores escolares e inspectores de enseñanza.

A partir de 1980, un amplio movimiento para la reformulación de la pedagogía, entre otras licenciaturas, adoptó el principio de la docencia como la base de la identidad profesional de los profesionales de la educación. A la luz de este principio, la mayoría de las instituciones adscribieron a los cursos de Pedagogía la formación de profesores de educación infantil, así como para las series iniciales de la enseñanza de primer grado (enseñanza primaria).

Con el fin de la dictadura, en 1985, y la consiguiente redemocratización del país, se llevó a cabo una nueva reforma, materializada en la Ley $n^{\circ}$ 9394, del 20 de diciembre de 1996. La importante movilización de educadores alimentó la expectativa de que, después del final del régimen militar, el problema de la formación docente en Brasil se abordaría mejor. Pero la nueva Lei de Diretrizes e Bases promulgada, después de varias vicisitudes, no cumplió con esta expectativa. Al presentar los Institutos Superiores de Educación y las Escuelas Normales Superiores como una alternativa a la pedagogía y otras licenciaturas, la Lei de Diretrizes e Bases marcó una política educativa dirigida a nivelar por abajo, porque los Institutos emergen como instituciones inferiores a las Universidades, al proporcionar una capacitación más liviana y barata a través de cursos cortos (SAVIANI, 2009). A estas características no eran inmunes las nuevas pautas curriculares del curso de pedagogía homologadas en abril de 2006.

En la actualidad, con el surgimiento de fuerzas políticas consideradas de extrema derecha, en un momento de conflictos y de desacuerdos políticos, la educación está en suspenso. Se anuncia una nueva reforma en la Educación, pero como no hay diálogo entre el gobierno y los intelectuales y trabajadores de la Educación, no podemos saber qué medidas se pueden adoptar, qué nuevos modos de formación docente pueden surgir. A pesar de la oscuridad del momento histórico, discutimos, a continuación, modelos didácticos y pedagógicos para la formación de docentes, que pueden servir como elementos facilitadores de la práctica pedagógica. 


\section{Formación de educadores: aspectos pedagógicos y didácticos generales}

Generalmente se distingue entre formación inicial y formación permanente de los profesionales de la educación, aunque ambas son complementarias. Si bien la primera se refiere a los programas básicos teórico-prácticos que preparan a los futuros educadores para desempeñar adecuadamente sus funciones $y$, la segunda, a la que acontece durante el ejercicio profesional, en gran medida para responder con éxito a los retos que surgen en una sociedad cambiante.

Nuestras Facultades de Educación-Centros de Educación se encargan de la formación inicial de los educadores, una preparación que debe equilibrar los aspectos teóricos y los prácticos y que, asimismo, ha de adaptarse a los rápidos cambios que se producen en la sociedad. Si pensamos, en la atención a la diversidad, se constata, según se desprende de trabajos como el firmado por Figueredo y Ortiz (2017), que los diseños curriculares analizados en Andalucía (España), por ejemplo, sobre formación inicial inclusiva del profesorado de Educación Primaria recogen indicaciones sobre la diversidad cultural, pero dichas referencias son escasas. Además, no se aprecia planificación ni coordinación en lo que se refiere al proceso de adquisición de competencias interculturales, ni se detecta un tratamiento transversal de las mismas a lo largo de los planes de estudio.

En el caso del profesorado de Educación Secundaria, el estudio realizado por Serrano y Pontes (2016) a partir de una muestra de más de 350 estudiantes, en España, todos ellos futuros docentes, permitió conocer que sus demandas formativas tienen que ver con: aplicaciones educativas de las nuevas tecnologías, pedagogía para la convivencia en el centro y el aula, programación educativa y diseño del currículo, atención a la diversidad y a las necesidades especiales, técnicas de dinámicas de grupo, psicología del desarrollo, uso didáctico de esquemas y mapas conceptuales, orientación educativa y acción tutorial, desarrollo de la autoridad académica, superación de situaciones de estrés profesional, técnicas e instrumentos de evaluación y desarrollo cognitivo del alumnado. Estos resultados revelan que los futuros profesores tienen diferentes necesidades que van más allá del ámbito disciplinar, pues se localizan sobre todo en el campo de la preparación psicopedagógica y didáctica. 
Investigaciones como las citadas permiten detectar algunas necesidades formativas del profesorado de los primeros niveles de la enseñanza y, al mismo tiempo, puede servir de referencia para enriquecer los planes de formación inicial, así como para organizar el diseño de la formación docente permanente, llamada a completar la preparación de los educadores, con arreglo a sus concretas necesidades y a las demandas sociales.

Datos aún más recientes obtenidos de la Organización para la Cooperación y el Desarrollo Económicos (OCDE, 2019) a partir del Estudio Internacional sobre Enseñanza y Aprendizaje (TALIS), revelan que en España únicamente el $48 \%$ de los docentes han estudiado durante su formación inicial los contenidos propios de sus materias, la didáctica específica y general y la práctica en el aula, un porcentaje que es bastante más bajo que el promedio obtenido por los países y economías de la OCDE que participan en TALIS y que representa un $79 \%$.

La formación permanente, por su parte, encuentra su razón de ser en el avance de los conocimientos y en la necesidad de responder adecuadamente a los desafíos que plantea una sociedad crecientemente compleja. La evolución científica, tecnológica, pedagógica y social exige a profesores y 12 educadores sociales en ejercicio un proceso formativo continuado de carácter integral y que, por tanto, excede el ámbito cognitivo, relativo a los conocimientos disciplinares. La formación de educadores, inicial y permanente, es una actividad pedagógica que reclama creciente atención y que no se circunscribe al ámbito técnico. Puede afirmarse que el perfeccionamiento profesional tiene incidencia en el desarrollo personal y, con carácter general, todo educador precisa de modo continuo enriquecimiento cognitivo, aptitudinal/competencial y valoral. Hablamos, en efecto, de unidad formativa en la que asumen mucha importancia los conocimientos de los profesionales en sus respectivas áreas; saberes de índole disciplinar, pero también didáctica que posibilitan la mejor educación. Desde una óptica amplia y abierta, el quehacer formativo de los educadores, sin soslayar las singularidades, debe comprometerse con actividades como enseñar, orientar, motivar, investigar, innovar, programar, mediar, optimizar, acompañar, gestionar, coordinar, organizar y un largo etcétera.

Las aptitudes o, quizá mejor, las competencias, dado que gozan actualmente de amplio reconocimiento en el mundo educativo, aunque su conceptualización sea compleja, permiten en cierto modo formalizar las actuaciones que se esperan de los profesionales a partir de criterios consensuados 
y consistentes. Su concreción responde a las demandas de la situación profesional a la que la formación se dirige. Y sin que invalidemos cuanto queda dicho sobre los conocimientos, no es extraño manejar una noción integradora de competencia, en la que se incluirían aspectos cognitivos (conocimientos, conceptos, "saber"), prácticos (destrezas, procedimientos, "saber hacer o aplicar") y actitudinales/axiológicos (disposiciones, valores, "saber estar y ser"). Desde este enfoque, algunas de las competencias de los profesionales de la educación guardan relación con el conocimiento especializado, con la comunicación y la relación interpersonal, con el análisis y la síntesis, con la reflexión etc.

La identificación de competencias nos ayuda a establecer diseños formativos para los educadores a nivel preprofesional y durante el ejercicio de la carrera. Está fuera de toda duda la importancia que las prácticas realizadas en instituciones educativas tienen durante la formación inicial. El acercamiento a la realidad profesional en variedad de escenarios complementa la preparación recibida en las aulas universitarias, pues brinda necesarias oportunidades de observación, comprobación, actuación y, en definitiva, de aprendizaje.

En cuanto a la promoción de los valores en el proceso formativo puede ser una cuestión controvertida, pero deber recordarse que son inherentes a la genuina educación. En todo proceso educativo se descubren valores, aunque sea de modo implícito, y en toda sociedad los valores dan cuenta de la auténtica vida humana plural, relacional y libre. La senda compartida de formación, inicial y continua, que cada profesional está llamado a recorrer no puede hacerse si se prescinde de los valores, sin los cuales resultará muy complicado, cuando no imposible, su fomento en los educandos. Por ello, los programas de formación de educadores deben contar con esa cimentación valoral impulsora de intersubjetividad, de cohesión, de comunicación, de vida en común, de convivencia, de ambiente educativo, para posibilitar el desarrollo saludable de la personalidad, tanto del educador como de los educandos, y, por ende, la mejora de la comunidad.

La formación permanente de los educadores no siempre recibe el reconocimiento que merece. A menudo depende de la motivación del propio profesional $y$, tanto si se realiza individualmente como en grupo, debe tener en cuenta la dimensión comunitaria de la educación. Ni el educador ni la institución en que trabaja son islas, así que tampoco el proceso formativo puede considerarse al margen de la sociedad. Por supuesto, cabe incluir entre las vías 
de desarrollo profesional la lectura, una actividad autónoma, no individualista, que proporciona una oportunidad de acrecentamiento en este mundo asediado por imágenes a la que no se debe renunciar, menos aún los profesionales de la educación. Otro canal formativo son los cursos de temática variada realizados en la institución o en otras entidades públicas o privadas, adaptados a las necesidades, intereses y circunstancias del personal educador. No puede faltar tampoco la reflexión sobre la propia acción educativa, en la línea de lo planteado por Schön (1992), ni el diálogo abierto y respetuoso con los colegas, encaminado a generar conocimiento, a compartir experiencias y prácticas, a fortalecer la conciencia y la cohesión grupal, a analizar situaciones y problemas, a buscar soluciones y a mejorar colaborativamente la educación, una comunicación formativa que queda enriquecida cuando se abre a los educandos, a las familias, a la realidad del centro de trabajo, a otras instituciones y a la sociedad en su conjunto.

Teniendo presente lo señalado hasta aquí, recordamos las desemejanzas y la complementariedad de la formación inicial y permanente, la importancia de singularizar el proceso formativo a las distintas profesiones educativas y la necesidad de adaptar dicho proceso a las demandas de nuestra

14 sociedad crecientemente compleja.

\section{Modelos en la formación de profesionales de la educación}

Aunque se habla en ocasiones de tendencias, orientaciones, perspectivas, enfoques, tradiciones, incluso de paradigmas formativos, optamos aquí por referirnos a modelos, porque queremos resaltar con dicho término que se trata de esquemas teóricos de alcance práctico, es decir, de representaciones que, en este caso, proporcionan claves para la mejora de la formación de los educadores.

Aunque hay pluralidad de modelos, los más destacados son cuatro: académico, técnico, personalizado y hermenéutico-reflexivo, sobre los que ofrecemos algunas notas. Estos modelos responden a ópticas pedagógicas diferentes que se proyectan sobre la formación de los profesionales de la educación. Proceden de tradiciones diferentes aunque no tienen por qué ser incompatibles. De un modo u otro, estos modelos, deudores del pasado, combinan teoría y práctica e impactan en toda la educación. Es indispensable 
reconocer la importancia que estas diferentes tradiciones en la formación de profesionales tienen en nuestros sistemas educativos y hacer lo posible para contextualizar dicha preparación según las singularidades históricas de cada escuela y casa sociedad, siempre con la vista puesta en los muchos desafios que se presentan em el horizonte:

\section{Modelo académico}

Este modelo formativo fundamentado en una racionalidad sapiencial enfatiza el rol de los educadores como intelectuales. Es una formación centrada en los respectivos ámbitos disciplinares, en la que asumen mucha importancia los contenidos, al tiempo que la preparación pedagógico-didáctica es considerada secundaria. Para esta tradición, el conocimiento científico en el propio campo profesional está llamado a completarse con las prácticas educativas en alguna institución.

La imagen del educador como un sabio nos sitúa ante un profesional que antaño, en la institución escolar, asumía todo el protagonismo, sin favorecer la interacción. Era una enseñanza vertical y a veces autoritaria que estampaba en la mente del educando, mediante un aprendizaje meramente memorístico, nada significativo, abundantes contenidos.

Esta corriente academicista, tradicional, puede resultar demasiado transmisiva, reproductiva y aun elitista. Al respecto, como recogen Liston y Zeichner (2003), a partir de distintas fuentes, algunas de las críticas recibidas tiene que ver con la inadecuada preparación de educadores para trabajar en áreas desfavorecidas y con gran diversidad cultural, algo que pretendió enmendarse, por ejemplo en los años 60 y 70 en EE.UU., mediante la introducción en los planes formativos de perspectivas centradas en la diversidad cultural.

\section{Modelo técnico}

Desde este modelo, cimentado en la racionalidad instrumental, se considera que el educador es un técnico, poseedor de los oportunos principios, conocimientos y prácticas que le permiten desenvolverse competentemente en 
entornos educativos. Es un especialista que ha de aplicar de modo eficiente el saber científico recibido durante el proceso formativo.

Uno de los enfoques representativos de este modelo es la Formación de Profesionales basada en Competencias. Con arreglo a este planteamiento, que tiene décadas de recorrido y llega con mucha fuerza hasta nuestros días, se trata de adquirir destrezas aplicables en entornos escolares o socioeducativos y previsiblemente vinculadas a los objetivos que se quieren alcanzar. Aun cuando esta orientación no es ajena en su origen al positivismo y a la rendición de cuentas, ha ido incorporando nuevas influencias y se ha hecho más versátil. Tal es la fuerza de este enfoque que numerosos países lo están incorporando, a pesar de las críticas que ha recibido relativas a la imprecisión y controversia en torno al término 'competencia', a la subordinación del modelo a la productividad, a la insuficiente consideración de los profesionales de la educación, al descuido de una educación inclusiva, etc., según puede advertirse en trabajos como el compilado por Gimeno (2008). Este mismo autor (GIMENO, 2008), por ejemplo, nos recuerda que el concepto de competencia ha estado muy arraigado en el mundo de la formación laboral para referirse al tipo de acciones que deben ejercerse en un determinado puesto de trabajo, para delimitar

16 las responsabilidades de quien lo desempeñe, para precisar las cualidades y destrezas de quienes quieran acceder a él, así como para disponer de criterios de valoración de la idoneidad de lo realizado.

Así pues, cabe decir que trasvasar este modelo al ámbito de la formación de profesionales en aras de la eficacia puede resultar utilitarista, pero obviaría el sentido profundo de la educación, su dinamismo, su compromiso humanizador, su carácter integral.

\section{Modelo personalizado}

Es un modelo humanista que parte de la consideración del profesional como persona, con toda su integridad, en una concreta situación sociohistórica y cultural, comprometida con la transformación positiva de la realidad. Congruente con la racionalidad humanista, es un proceso formativo que enfatiza la apertura, la singularidad, la autonomía en un marco de cooperación/ colaboración, la reflexión, la creatividad, la comunicación y las relaciones interpersonales, la convivencia y la unidad ante la variedad de tareas y funciones 
que el educador está llamado a realizar. Los programas de formación inicial y permanente de educadores adquieren sentido cuando todos sus elementos se hallan cohesionados y contribuyen adecuadamente al fin previsto. Con carácter general, la formación de educadores ha de contemplar a un tiempo la vertiente técnica y la vertiente humana. Esta integración formativa armónica, que no se circunscribe a lo establecido en los programas, se profundiza igualmente con la unidad entre teoría y práctica.

Ya hace años señalaba García Hoz (1996) que dado que cualquier programa educativo incluye conocimientos, aptitudes y valores, la formación de educadores debe hacerse cargo igualmente de los tres aspectos: conocimientos necesarios para ejercer la correspondiente función educadora; aptitudes para llevar a cabo las tareas concretas que dicha función exige, y valores, porque en ellos se condensa el sentido de una acción verdaderamente educativa. A fin de cuentas, todo lo que se haga o diga en un programa de formación de educadores influye en los conocimientos, en las aptitudes, en los valores de los profesionales y, por supuesto, en ellos en cuanto personas.

Esta formación personalizada, en la que se da importancia a los aspectos cognitivos, afectivos y éticos, al autoconcepto y a la maduración, implica que el educador pueda autorrealizarse merced al desarrollo del proyecto de vida, del que la profesión es parte significativa. En todo el proceso formativo la cultura, la sociedad, la historia, la actitud dialógica y las relaciones humanas son claves para impulsar la mejora personal, profesional y social.

El modelo personalizado también ha recibido críticas, algunas basadas en una supuesta desatención formativa de los aspectos relacionales del educador y de su compromiso de transformación social, quizá consecuencia de una visión reducida de lo que la personalización es. Este concepto, en su sentido verdadero y profundo, no renuncia en modo alguno a los objetivos relacionales y sociales que todo proceso formativo alberga.

\section{Modelo hermenéutico-reflexivo}

Este modelo de formación, cuyos pilares se descubren en la racionalidad indagadora y práctica, rechaza el mecanicismo de los planteamientos técnicos, positivistas. Busca sobre todo que el profesional interprete la realidad a partir de la indagación. Rechaza las prescripciones generales y presta 
atención a la situación, a la valoración e implicación de los profesionales, a las relaciones que establecen entre sí, etc. El modelo hermenéutico-reflexivo, a diferencia del modelo técnico, se preocupa más por las motivaciones, las experiencias/vivencias, los sentimientos y los sentidos/significados de los profesionales que por la eficiencia y la evaluación de competencias. Podría decirse, a partir de Marcelo (1999), que la reflexión como aspecto clave en la formación de educadores se traduce en un alejamiento de planteamientos estáticos, incluso reproductivos, respecto al currículum, la planificación, el conocimiento, la enseñanza, la intervención y la profesión en su conjunto. Si tomamos como referencia a Pumares (2012), la formación de los educadores de acuerdo a este modelo debe tener en cuenta: la investigación educativa de índole cualitativa; el fomento de la reflexión y la interpretación de la realidad, más que la mera transmisión de contenidos; la elaboración de materiales a partir de variedad de fuentes y en función de los intereses, expectativas, necesidades y situaciones; el papel activo de los profesionales, responsables de su aprendizaje y desarrollo; el impulso de un liderazgo democrático; la búsqueda de la participación y el consenso en las instituciones educativas -escolares o no-, con reparto de poder, delegación de funciones y cultivo de la libertad; la 18 evaluación cualitativa; los procesos más que los resultados; la responsabilidad compartida y multicausal, con revisión crítica de las distintas actuaciones y de todo el proceso.

Es un modelo que insiste en la necesidad de que los profesionales reflexionen sobre las actividades educativas que realizan o han de realizar. Se parte de la base de que la actuación de los educadores será más innovadora y mejor si hay un compromiso reflexivo a nivel individual y colectivo, en el que se analizan las circunstancias educativas, sociales, históricas, etc. Este modelo, que contempla al educador como profesional reflexivo e incluso como un artista, puede adentarse por una senda sociocrítica-reconstruccionista, distinguida por la actuación emancipadora, que exige necesariamente una concienciación/concientización del propio mundo vital y de las contradicciones y constricciones del discurrir humano-social, por ejemplo, los intereses sectoriales, la manipulación, la opresión etc. Para esta perspectiva sociocrítica, de equivalente racionalidad subyacente (crítica, reconstructiva, liberadora), los profesionales de la educación han de ser activistas que, lejos de conformarse con interpretar o comprehender las situaciones, intervienen para alcanzar prácticas educativas y sociales más justas. 
Precisamente, algunas de las críticas vertidas sobre el modelo hermenéutico-reflexivo proceden de sectores reconstruccionistas, que consideran social y pedagógicamente insuficientes los planteamientos indagadores e interpretativos y enfatizan el carácter emancipador de la educación y la profesionalización. Por su parte, también esta corriente sociopolítica ha recibido críticas sobre todo por los que señalan que una formación de educadores como la propugnada está ideologizada y puede ser mera herramienta al servicio de la política.

\section{Consideraciones finales}

En la Primera República, la instrucción pública alcanzó una importancia primordial, al menos en el nivel del discurso, en la consolidación de un nuevo proceso civilizador, por ser considerada como el elemento racional, modernizador y organizador de la sociedad, haciéndola más justa e integrada con los parámetros modernos de progreso social y, quizás, económico. Este discurso estuvo cargado de ideas liberales y encontró su mayor expansión con el advenimiento de la República y su ideología positivista.

Así, evaluamos que la República Brasileña hasta 1930 representó un período de transición entre un mundo político-cultural-económico derivado de una sociedad de gobierno imperial, con sus propios hábitos y cultura, para ingresar a una sociedad moldeada en bases republicanas y económicamente respaldada por bases capitalistas más avanzadas, aunque en el marco del capitalismo. La llamada Revolución de los 30 permitió cambios más rápidos en la educación, con las reformas de Francisco Campos (1931) y luego la reforma de Capanema (1942, 1943 y 1946), a cultura e incluso la economía, que consolidaron el nuevo proceso de civilización republicana.

Así pues, tras realizar un repaso histórico de la educación en Brasil, en el que sobresalen, según los momentos, ciertos rasgos, legislaciones e instituciones, se destaca el interés y la intervención de los poderes públicos tanto en la educación, considerada el verdadero motor de progreso, como en la formación del profesorado, eje del proyecto civilizador. Las diversas iniciativas y reformas educativas, principalmente estatales, aunque también dejó su huella la acción educativa y evangelizadora de la Iglesia, revelan avances en el ansiado proceso modernizador, pero manifiestamente insuficiente, 
especialmente en lo que se refiere a los sectores más desfavorecidos de la población. Las orientaciones educativas tenían sobre todo un carácter práctico y ocupacional, especialmente para la mayor parte de la población. La formación superior respondía a una óptica más académica y científico-técnica, pero quedaba en gran medida restringida a los grupos más privilegiados.

En lo que se refiere a la formación del profesorado, se descubre, en general, su insuficiencia, acaso consecuencia de la inveterada desconsideración pedagógica y social de esta profesión, infravaloración que llega hasta nuestros días, según se advierte también en el terreno salarial. La preparación de profesores en Brasil respondió en muchos aspectos a un modelo pedagógico académico, tradicional, eminentemente transmisivo, que posteriormente se trasladaba y reproducía en las aulas, con alumnos en el papel de meros receptores de conocimientos, a menudo endeblemente aprendidos con anterioridad por los propios docentes. El sesgo moralizante y religioso que muchas veces tenía esta formación de profesores, dio paso, con el discurrir del tiempo, y por influencia de tendencias internacionales que llegan hasta la actualidad, a procesos formativos de carácter técnico, propios de un modelo en el que no es extraño destacar la dimensión competencial, el logro de resultados y la 20 eficacia.

El repaso histórico de la formación del profesorado y de la configuración del sistema educativo en Brasil nos ofrece pistas sobre lo que acontece en la actualidad, por cierto bastante desconcertante si nos atenemos a las medidas y a los recortes que en materia de educación está adoptando el actual gobierno.

Aunque los fundamentos pedagógicos en lo que a formación docente se refiere puedan variar, permanecen en el país los problemas relacionados con dicha formación, por ejemplo, en aspectos metodológicos, organizativos, didácticos y relacionales, al igual que la insuficiencia de recursos. De un modo u otro, cualquier disfunción de este tipo tiene un impacto negativo en el propio profesional, en la institución educativa, en el conjunto de la sociedad y, desde luego, en los alumnos, verdaderos destinatarios y protagonistas de la educación. La superación de muchas de estas dificultades, algunas recientemente destacadas por la OCDE (2019), no parece encontrarse en las tradiciones exclusivistas o en la idealización de modelos, sino en la colaboración, apertura e interrelación de elementos y agentes que intervienen en el quehacer formativo, en un marco de inversión razonable en educación. 
Ciertamente, cada modelo alberga de modo más o menos explícito una racionalidad/epistemología, unos valores, unos principios y unas concepciones acerca de la persona, del profesional, de la educación y de la sociedad, pero dado que la actividad profesional de los educadores se desarrolla en contextos complejos, podría ser conveniente una visión amplia e integradora, en la que se adoptase una combinación de aspectos de los distintos modelos. Quizá una actitud así, advertida en la preparación inicial y continua del profesorado, sea la única opción para que de una vez por todas la formación docente, con los oportunos recursos, tenga la consideración que se merece, permita a los profesionales de la educación enfrentarse con éxito a los desafíos cotidianos en las aulas y, en definitiva, cumpla su trascendente papel pedagógico y social.

\section{Referências}

AZEVEDO, Fernando de. A cultura brasileira: introdução ao estudo da cultura no Brasil. Brasília: Editora UNB, 1996.

BRASIL. Lei de 15 de outubro de 1827. Manda crear escolas de primeiras letras em todas as cidades, villas e logares mais populosos do Imperio.Brasilia/DF. Disponível em: https:// www2.camara.leg.br/legin/fed/lei_sn/1 824-1899/lei-38398-15-outubro-1827-566692-publicacaooriginal-90222-pl.html. Acesso em: 15 ago.2019.

FERRARO. Alceu Ravanello. História inacabada do analfabetismo no Brasil. São Paulo: Cortez, 2009.

FIGUEREDO, Victoria; ORTIZ, Luis. Formación inicial del profesorado para la inclusión de la diversidad cultural.Revista Digital de Investigación en Docencia Universitaria, Lima, v. 11 , n. 1, p. 38-61, ene./jun. 2017.

GARCÍA HOZ, Víctor. "Vocación pedagógica universal". En GARCÍA HOZ, Víctor et al.: Formación de profesores para la educación personalizada, pp. 19-45, Madrid, Rialp. 1996.

GIMENO SACRISTÁN, José (Comp.). Educar por competencias, ¿qué hay de nuevo? Madrid: Morata, 2008.

GIMENO SACRISTÁN, José. "Diez tesis sobre la aparente utilidad de las competencias en educación". En GIMENO SACRISTÁN, José (Comp.). Educar por competencias, ¿qué hay de nuevo? Madrid: Morata, 2008. 
IBGE. Instituto Brasileiro de Geografia e Estatística. Metodologia do Censo Demográfico 2000. Rio de Janeiro: IBGE, 2003. Disponível em: hitp://goo.gl/UvlwF. Acesso em: 10 ago. 2019.

LIMA, Nestor dos Santos. Um século de ensino primário. Natal: Tipografia da República, 1927.

LISTON, Daniel Patrick; ZEICHNER, Kenneth. Formación del profesorado y condiciones sociales de la escolarización. Madrid: Morata, 2003.

MARCELO, Carlos. Formación del profesorado para el cambio educativo. Barcelona: EdicionesUniversitarias de Barcelona, 1999.

MOACYR, Primitivo. A Instrução e o Império (Subsídios para a História da Educação no Brasil) - 1854-1889. São Paulo: Companhia Editora Nacional, 1936.

MENDONCCA, Ana Waleska Pollo; CARDOSO, Tereza Maria Rolo Fachada Levy. A gênese de uma profissão fragmentada. Revista Brasileira de História da Educação, Campinas, v. 7, n. 3, p. $31-52$, set./dez. 2007.

OCDE. Organização para a Cooperação e Desenvolvimento Económico. Resultados de TALIS, 2018. Nota país. España. Disponível em: http://www.oecd.org/education/talis/ 22 TALIS2018_CN_ESP_es.pdf. Acesso em: 15 ago. 2019.

PAIVA, Marlúcia Menezes. Educação e modernidade: os anseios republicanos no Rio Grande do Norte. 2009 (Manuscrito).

PUMARES, LUis. I. El desarrollo profesional docente y los modelos de racionalidad. En Gonzalo Virginia: Desarrollo profesional de docentes y educadores, Madrid, Los libros de la Catarata, 2012.

SCHÖN, Donald. La formación de profesionales reflexivos. Hacia un nuevo diseño de la enseñanza y el aprendizaje en las profesiones. Paidós: Barcelona, 1992.

SAVIANI, Dermeval. Formação de professores: aspectos históricos e teóricos do problema no contexto brasileiro. Revista Brasileira de Educação, Rio de Janeiro, v. 14, n. 40, p. 143155, jan./abr. 2009.

SERRANO, Rocío; PONTES, Alfonso. Necesidades formativas en el futuro docente de Educación Secundaria. Revista Educativa Hekademos, Espanha, v. 9, n. 21, p. 74-82, dec. 2016. 
SILVA, Francinaide de Lima. O Grupo Escolar Modelo Augusto Severo: história e prática pedagógica (Natal/Rio Grande do Norte, 1908, 1920). 2009. 170 f. Dissertação (Mestrado em Educação) - Programa de Pós-Graduação em Educação, Universidade Federal do Rio Grande do Norte, Natal, 2009.

Prof. Dr. Valentín Martínez-Otero Pérez

Universidad Complutense de Madrid

Departamento de Estudios Educativos

Programa de Pós-Graduação em Educação

Grupos de Pesquisa Psicosociobiología de la Violencia: educación y prevención

E-mail:valenmop@edu.ucm.es

ORCID ID: https:/ / orcid.org/0000-0003-3971-7204

Profa. Dra. Marlúcia Menezes de Paiva

Universidade Federal do Rio Grande do Norte

Departamento de Fundamentos e Políticas da Educação

Programa de Pós-Graduação em Educação

Grupo de Pesquisa Fundamentos da Educação e Práticas Culturais

ORCID ID: https/ / orcid.org/0000-0002-1 123-342X

E-mail:mmarlupaiva3@gmail.com

Recebido 9 set. 2019

Aceito 7 nov. 2019 